\title{
Competitividad, planeación estratégica y Tecnologías de Información en pequeños restaurantes y hoteles de Villahermosa
}

\author{
Gerardo Arceo Mohenoํㅜ Eric Ramos Méndez ${ }^{2}$ y Carlos Mario Flores Lázaro ${ }^{3}$ \\ ${ }^{1}$ Universidad Juárez Autónoma de Tabasco, División Académica de Ciencias y Tecnologías de Información \\ Villahermosa, Tabasco, México, ericarceo@hotmail.com, Carretera Cunduacán-Jalpa KM. 1 Col. La Esmeralda, \\ Cunduacán, Tabasco (+52) 9143360870 \\ ${ }^{2}$ Universidad Juárez Autónoma de Tabasco, División Académica de Ciencias y Tecnologías de Información \\ Villahermosa,Tabasco, México, ericramos@hotmail.com, Carretera Cunduacán-Jalpa KM. 1 Col. La Esmeralda, \\ Cunduacán, Tabasco (+52) 9143360870 \\ ${ }^{3}$ Universidad Juárez Autónoma de Tabasco, División Académica de Ciencias Económico-Administrativas \\ Villahermosa, Tabasco, México, carlos.flores@ujat.mx, Av. Universidad S/N, Zona de la Cultura, Villahermosa, \\ Tabasco (+52) 9933581500
}

Información del artículo revisado por pares

Fecha de aceptación: junio-2021

Fecha de publicación en línea: diciembre-2021

DOI: https://doi.org/10.29105/vtga7.1-119

\section{Resumen}

En el mundo actual, el competir entre las empresas cumpliendo con las expectativas y necesidades de los clientes se ha vuelto obligatorio. Ante este contexto, la planeación estratégica debe desempeñar un papel incuestionable. Adicionalmente se menciona que la literatura señala que el uso estratégico de las tecnologías de información (TI) inciden en la competitividad $\mathrm{y}$, en consecuencia, en la supervivencia empresarial. En la presente ponencia se presentan los resultados obtenidos de un estudio hecho a pequeños restaurantes y hoteles ubicados en la ciudad de Villahermosa, municipio de Centro, estado de Tabasco, el cual tuvo como objetivo conocer la situación de estas empresas en cuanto a la competitividad mostrada, su conocimiento y realización de estrategias y actividades de planeación, así como los usos proporcionados a las TI. El estudio fue descriptivo no experimental y se hizo uso del enfoque mixto, realizándose en 66 restaurantes y 78 hoteles. Para la recolección de datos se diseñó y usó un cuestionario con escala Likert. Entre los resultados se encontró que la gran mayoría de las empresas no conocen la planeación estratégica, por lo que muy pocas de sus actividades se llevan a cabo (de forma empírica). En cuanto a las TI, a pesar de la importancia dada por los encuestados, se encontró que los usos dados podrían no verse como estratégicos. Estos resultados, sin duda, están incidiendo en la competitividad.

Palabras clave: Competitividad, Hoteles, Planeación estratégica, Restaurantes, TI.
Abstract
In today's world, competing between companies meeting customer expectations and needs has become mandatory. In this context, strategic planning must play an unquestionable role. Additionally, it is mentioned that the literature indicates that the strategic use of information technologies (IT) affects competitiveness and, consequently, business survival. This presentation presents the results obtained from a study of small restaurants and hotels located in the city of Villahermosa, municipality of Centro, state of Tabasco, which aimed to know the situation of these companies in terms of competitiveness shown, their knowledge and implementation of strategies and planning activities, as well as the uses provided to IT. The study was descriptive and non-experimental and the mixed approach was used, being carried out in 66 restaurants and 78 hotels. For data collection, a questionnaire with a Likert scale was designed and used. Among the results, it was found that the vast majority of companies do not know strategic planning, so very few of their activities are carried out (empirically). Regarding IT, despite the importance given by the respondents, it was found that the uses given might not be seen as strategic. These results are undoubtedly affecting competitiveness.

Keywords: Competitiveness, Hotels, Strategic Planning, Restaurants, IT JEL: M1, M10, M15 y M19. 


\section{INTRODUCCIÓN}

No hay otra ciudad en México donde el agua sea esencia de su espíritu como la capital de Tabasco: la ciudad de Villahermosa. Las aguas de los ríos Grijalva y Carrizal, así como de las lagunas de las Ilusiones y el Negro, bañan sus riberas permitiendo la creación de hermosos parques y museos, como el Parque Tomás Garrido Canabal, el principal "pulmón verde" de la ciudad, donde la laguna de la Ilusiones ofrece paisajes de ensueño, así como el Parque-Museo La Venta, donde se encuentran albergadas las colosales cabezas olmecas halladas en la Zona Arqueológica de la Venta. Adicionalmente, la ciudad ofrece más atractivos turísticos, como lo es la llamada "Casa de los Azulejos", museo que muestra la historia de la ciudad; la Plaza de Armas, allende al río Grijalva y donde se encuentra el Palacio de Gobierno y la Iglesia de la Inmaculada Concepción, de fachada gótica; o bien, la Casa Carlos Pellicer Cámara, donde nació y vivió el poeta de los tabasqueños.

Por si fuera poco, la capital tabasqueña es popular por su cocina que toma como base ingredientes mayas y chontales que dan origen a platillos destacados en la gastronomía mexicana como el pejelagarto asado, tamalitos de chipilín, chirmol, el plátano en todas sus variantes, tortillas gruesas de maíz, etc.

Lo anterior muestra algo de lo mucho que ofrece la capital del "edén", el segundo núcleo administrativo y comercial de la región Sureste de México.

Pese a esta riqueza turística y cultural, la ciudad no se destaca como un destino preponderante o final de los turistas. Como Villahermosa es conocida como la entrada al "Mundo Maya" (Palenque, Mérida, Edzná, etc.), muchos turistas solo arriban de paso para dirigirse casi de inmediato a las zonas mencionadas.

Muchas de las personas que llegan a la ciudad podrían catalogarse como "turismo de trabajo" (derivado de la industria petrolera), mientras que los que llegan por turismo de placer provienen de nuestro propio país (regional en su mayor parte). De acuerdo a lo antes expuesto, se está desaprovechando el gran potencial turístico que tiene la ciudad (y el estado de Tabasco en general) $\mathrm{y}$, por ende, los grandes beneficios económicos que conlleva.

En este desarrollo del turismo se deben replantear las oportunidades que existen, considerando los elementos que juegan un papel preponderante dentro de estas mismas, siendo parte de estos elementos los restaurantes y hoteles que existen y se ofertan en la ciudad.

Actualmente, se observa que el turismo de negocios que llega a la ciudad ocupan hoteles con servicios de la calidad de 3 estrellas o más (hoteles de cadenas tales como Hyatt, One, Fiesta Inn, etc.) y consumen en restaurantes firmemente establecidos (ya sea que estén establecidos cerca de los hoteles, como Rodizio, La Jangada, etc., o bien, restaurantes de cadenas como Vips, Toks, etc.), dejando de lado aquellos pequeños restaurantes y hoteles que, debido al desconocimiento de habilidades administrativas, no son conocidos fuera de la localidad originando una baja demanda de ellos $\mathrm{y}$, por ende, su mortandad y el desempleo que conlleva, y en este contexto, es pertinente mencionar que las PYMES tabasqueñas no se encuentran en el grado de competitividad que deberían (Mandujano et al., 2014).

Una empresa competitiva debe poseer aquellas capacidades que pueda permitirle acceder, permanecer y/o crecer en un sector ya constituido, el cual es susceptible de oponerse a esta entrada pudiendo crear conflictos con los objetivos de la empresa (Bueno, 1996).

En este ambiente tan competitivo, se hace necesario que la empresa cree ventajas competitivas que le permita diferenciarse de sus competidores. López (2007) indica que una gran ventaja competitiva se obtiene mediante un eficiente uso de las tecnologías de información (TI) y su significativa aportación a la gestión del negocio, lo cual es factible de alcanzar a través de la aplicación de un enfoque de sistemas que potencie a las personas para su uso óptimo, entendiendo así el sentido estratégico de la aplicación de estas tecnologías dentro de la organización. Laudon y Laudon (2008) resaltan que para el logro de niveles de eficiencia, productividad y competitividad satisfactorios, los administradores cuentan con los sistemas y las tecnologías de información como uno de los elementos de apoyo más relevantes.

Por su parte, Porter (1996) señala que la competitividad empresarial está definida por la productividad que demuestra al usar sus recursos humanos, económicos y materiales, requiriendo para esto una estrategia que vaya acorde a las metas y objetivos propuestos.

Ahondando en este tema, se podría decir que la estrategia se concibe como un proceso orientador que señala, principalmente, los objetivos de la organización, los recursos para alcanzarlos y las políticas y normas que guiarán la 
administración de tales recursos. Estas estrategias deben considerar su influencia en la conducta de los recursos humanos al interior de la organización y, por tanto, en su competitividad laboral para que se logre así la eficiencia organizacional.

Por supuesto que estas estrategias deben definirse considerando tiempo y espacio, además de los objetivos a cumplir; por tanto, su diseño debe ser hecho a través de la planeación estratégica.

La planeación estratégica deberá posicionar a la organización dentro de su entorno, moviendo de manera acorde los recursos internos con los objetivos, permeando toda la estructura organizacional hasta llegar a los niveles más operativos.

No puede pasarse por alta que la planeación estratégica debe ser un proceso continuo y permanente en el tiempo, teniendo cuidado de que la dirección de la organización aborde, con el menor riesgo posible, las condiciones que se puedan presentar en el futuro.

Por tanto, se puede decir que la planeación estratégica se basa en el análisis interno de la organización considerando el contacto con el exterior, lo cual debe resultar en el planteamiento de unos objetivos a largo plazo, viables y medibles, que deben materializarse en estrategias con indicadores y meta, las cuales deben redundar, de alguna manera, en la competitividad organizacional

Pese a la relevancia de la planeación estratégica para alcanzar la competitividad y el papel que desempeñan las TI en este proceso, considerando la región Sureste de México y particularmente la ciudad de Villahermosa, no se ha encontrado evidencia empírica que muestre como se lleva a cabo la planeación estratégica, los usos dados a las TI en esta planeación y los alcances competitivos que tienen los pequeños restaurantes y hoteles de la ciudad antes mencionada, por lo que se espera proveer de nuevas aportaciones teóricas mediante los resultados obtenidos en este estudio y mostrados en la presente comunicación.

Las preguntas de investigación que das origen a este trabajo son las siguientes: ¿Cómo se llevan a cabo las actividades de planeación estratégica? ¿Se usan las TI en estas actividades? y ¿Existe incidencia en la competitividad empresarial?

\section{MARCO TEÓRICO}

\subsection{Competitividad}

Muchas de las pequeñas empresas, dentro de las que se engloban las empresas objeto de este estudio, consideran que, por estar dirigidas a un sector particular y que usualmente es local o regional, no es imprescindible el hecho de ser competitivas y confían en sus variables de mercado que, de acuerdo a sus administradores, conocen y controlan. Estas organizaciones, a diferencia de las grandes empresas, no destacan por innovar sus actividades dentro de sus procesos (llámese de producción o comercialización).

En el pasado, las pequeñas empresas basaban su competitividad en la flexibilidad y eficiencia en cuestión de la entrega de sus productos en mercados relativamente estables y pequeños. Sin embargo, hoy en día las condiciones no son las mismas: las TI imperan en todos los ámbitos por lo que empresas de cualquier tamaño localizadas fuera del área geográfica de confort pueden llegar a ofrecer sus servicios y/o productos con una mejor relación calidad/precio. En el caso de los hoteles, los nuevos modelos de negocios propiciados por las TI han revolucionado el mercado (el más conocido es Airbnb). En este ámbito, se hace imperativo que las empresas diseñen y lleven a cabo estrategias que las puedan conducir a la competitividad cumpliendo con los requerimientos de sus clientes (Heredia, 2014).

Cabe señalar que la competitividad se ve como una diferencia positiva percibida por los clientes sobre la competencia la cual debe permanecer un tiempo perdurable. Cuando una empresa está mejor posicionada que sus competidores permitiéndole fidelizar a los clientes y defenderse contra fuerzas competitivas, se puede decir de que ésta cuenta con una ventaja competitiva.

Para Alarco (2011), la competitividad no es, por sí sola, un fin u objetivo, sino un poderoso medio para alcanzar elevados estándares de vida incrementando el bienestar social y que involucra elementos de eficiencia, productividad $y$ rentabilidad

\subsection{Planeación estratégica}

Al desenvolverse en un ambiente competitivo que sobrepasa las fronteras locales y nacionales, las pequeñas empresas se encuentran cara a cara con amenazas y riesgos (y también con oportunidades) que las deben impulsar a establecer sus propias estrategias al momento de enfrentarlos. Independientemente de su tamaño, estas empresas deben saber definir las estrategias que les permitan sobrevivir a lo largo del tiempo, comprometiéndose a llevarlas a cabo realizando aquellas acciones que requieren dicho 
cumplimiento.

La planeación, tal como lo señalan Robbins y Coulter (2014) es aquella función que considera la definición de los objetivos organizacionales, el establecimiento de las estrategias para alcanzarlos, así como el desarrollo de los planes para integrar y coordinar las actividades laborales.

Rodríguez (2005) señala que la planeación estratégica se define como aquel conjunto de planes organizacionales que regularán el comportamiento de una empresa con el propósito de que resulte en la obtención de ventajas competitivas logrando, entre otros objetivos, las preferencias de los clientes por sobre sus competidores. Toda empresa, independientemente de su tamaño, debe dar la importancia y el tiempo adecuado y suficiente para el establecimiento de estas estrategias, considerando los recursos y capacidades que poseen y que marcan la diferencia con sus competidores (Leyva et al., 2018).

Chiavenato (2011) indica que la relevancia de la planeación estratégica radica en la capacidad con que cuenta la empresa para poder realizar un análisis de su entorno y tomar decisiones acertadas en un mundo global.

Para David (2007), esta planeación se ve como el proceso por el cual los directivos de una organización prevén su futuro, por lo que desarrollan los procedimientos y operaciones necesarios para alcanzarlo. Esta visión del estado futuro de la empresa señala la dirección en que se deben dirigir las energías de todos los recursos de la organización.

Es importante resaltar que la planeación estratégica no representa la solución a todos los problemas a los que se enfrenta la empresa, pero sí que representa una herramienta necesaria para la toma correcta de decisiones si se utiliza en el modo y momento adecuados.

\subsection{Tecnologías de información}

Desde inicios de este siglo, vivimos dentro de una sociedad que los especialistas denominan "Sociedad del Conocimiento", la cual se caracteriza por el uso de las TI de una forma inteligente en prácticamente todas las áreas de acción del ser humano. Para acceder a esta sociedad, el hombre ha tenido que pasar por diversas etapas evolutivas que involucran la Revolución Industrial hasta llegar a la Revolución Digital, dada en la década de los 90 cuando irrumpen las nuevas tecnologías (de ese entonces) ligadas a la información y comunicación originando el surgimiento de la Sociedad de la
Información, caracterizada por un uso intensivo de las mismas

Una organización puede alcanzar ventajas competitivas a través del uso de las TI mediante la optimización del flujo de la información y reforma(s) de los procesos administrativos y/o de producción.

La literatura señala que las TI deben ser integradas en los procesos organizacionales de las empresas debiendo ser un impulsor de la innovación, de tal manera que se conviertan en un instrumento real para alcanzar la competitividad suficiente para lograr una sobrevivencia a largo plazo a través de obtención de calidad en productos, eficiencia en los servicios, disminución en los tiempos de entrega, reducción de costos, entre otros (León y Valenzuela, 2014). En este sentido, no es de extrañar que muchas empresas hagan sobreesfuerzos para incorporar en su estructuras áreas de desarrollo informático dentro de ellas; sin embargo, se señala que muchas de estas empresas se limitan a llevar a cabo este plan simplemente como imitación, sin considerar las propias ventajas estratégicas que pudiesen obtener y olvidando que deben estar atentas a los factores de su entorno para hacer un análisis determinante del mismo y, de esta manera, hacer una implementación adecuada de las TI.

El uso de las TI se debe ver como un impulsor para que las empresas implementen estrategias que las conduzcan a la mejora en su eficiencia y productividad. García y Hernández (2017) resaltan la fuerte relación entre la innovación y el uso de las TI, al conducir a las empresas hacia el encuentro de oportunidades y la ampliación de sus ventajas competitivas en el mercado global, lo que le permitirá impactar en el rendimiento empresarial.

\subsection{Pequeñas restaurantes y hoteles}

Para Vásquez (2012), los restaurantes son establecimientos comerciales que sirven a los clientes comida y bebida para ser consumidas en el mismo local.

Velázquez (2009) señala que los restaurantes pueden clasificarse en:

- De lujo (cinco tenedores)

- De primera (cuatro tenedores)

- De segunda (tres tenedores)

- De tercera (dos tenedores)

- De cuarta (un tenedor)

Los restaurantes estudiados corresponden a la última categoría, los cuales cuentan con las siguientes características:

$\checkmark$ Comedor independiente de la cocina.

$\checkmark$ Cristalería sencilla, cubertería inoxidable, 
vajilla de loza y vidrio, servilletas de tela o papel.

$\checkmark$ Servicios sanitarios decorosos.

$\checkmark$ Personal perfectamente aseado

$\checkmark$ Carta sencilla.

En cuanto a los hoteles, Martínez (2013) los define como edificios planificados y acondicionados para otorgar servicio de alojamiento a las personas temporalmente. Por su parte, la Secretaría de Turismo (SECTUR) (2016) menciona que un hotel está catalogado como aquél que se proporciona en un establecimiento con un mínimo de 10 habitaciones que se ha instituido para proveer básicamente alojamiento, alimentación y los servicios complementarios demandados por el turista.

De acuerdo a la propia SECTUR (2016), los hoteles se clasifican de acuerdo a los servicios proporcionados por ellos desde Gran Turismo, seguido de 5 estrellas hasta llegar a 1 estrella. En el presente estudio se consideraron hoteles de 2 y 1 estrellas, los cuales se caracterizan por:

- contar con cafetería a horas determinadas

- limpieza en la habitación

- cambio de blancos y toallas diario

- mobiliario sencillo

- personal de servicio y atención al huésped limitado a la atención de limpieza en cuartos y recepción de huésped

Cabe resaltar que los administradores de las pequeñas empresas, como las consideradas en este estudio, representan el más débil eslabón de la cadena productiva y de servicios, principalmente ocasionado por la falta de preparación formal administrativa, ausencia de emprendimiento, la carencia de sistemas de planeación y control eficaces, así como la falta de propias TI para desarrollar sus actividades empresariales (Pizzi y Brunet, 2013).

\section{MÉTODO}

\subsection{Enfoque y tipo de investigación}

Este estudio es de carácter descriptivo al buscar especificar propiedades, características y rasgos importantes de las variables sometidas a análisis. De acuerdo a este tipo de investigación, se busca la recopilación de información para que mediante su análisis e interpretación se proceda a la descripción de los diferentes ítems que constituyen las variables de estudio.

Asimismo, de acuerdo al nivel de profundidad de análisis, fue una investigación no experimental, ya que las variables involucradas (competitividad, planeación estratégica y TI) no han sido susceptibles, de acuerdo a su naturaleza, a ser sometidas a experimentación.

El enfoque de investigación usado fue el mixto, al incluir el enfoque cualitativo $y$ cuantitativo. Hernández et al. (2010) describen que el método mixto representa un conjunto de procesos sistemáticos, empíricos y críticos de investigación e implica la recolección y el análisis de datos cuantitativos y cualitativos, así como su integración y discusión conjunta para realizar inferencias, producto de toda la información recabada, para lograr un mayor entendimiento del fenómeno bajo estudio.

\subsection{Estrategia metodológica}

La presente investigación parte de una base de conocimientos previos sobre la realidad estudiada hasta llegar a conocer la problemática real en una población determinada, a partir de la cual se plantearon soluciones probables.

La estrategia metodológica se menciona a continuación:

- Primera Fase: Revisión de literatura relacionada con los temas de estudio procedente de fuentes secundarias (libros, artículos especializados, tesis y páginas Web).

- Segunda Fase: Identificación de la población de estudio y determinación de la muestra estadística.

- Tercera Fase: A partir de la literatura revisada, se procedió a la elaboración del instrumento de recolección de datos.

- Cuarta Fase: Aplicación del instrumento de recolección de datos a la población de estudio determinada (fuente primaria).

- Quinta Fase: Organización y tabulación de los datos obtenidos lo que permitió su representación en términos cuantitativos a través de tablas y gráficas

- Sexta fase: Análisis e interpretación de los datos.

\subsection{Población y tamaño de la muestra}

De acuerdo a información proporcionada por la Cámara Nacional de la Industria de Restaurantes y Alimentos Condimentados (CANIRAC) Delegación Tabasco y la Asociación Mexicana de Hoteles y Moteles de Tabasco, A.C, en Villahermosa se encuentran 79 pequeños restaurantes y 97 hoteles que cumplen con los requerimientos solicitados para este estudio.

Se hace mención que la información de la mayoría los restaurantes y hoteles que constituyeron la población de estudio se encuentra en páginas electrónicas informativas 
como son Sección Amarilla, Fourquare, Pueblo América, Mx.Empresa, Directorio México, Foro-México, Infolugares, Directorio Tabasco, Guialis e Infoisinfo, señalándose que es información básica, tal como dirección, código postal, número telefónico, horario de servicio y mapa de localización. Es pertinente mencionar que estas páginas electrónicas, conocidas como páginas Web estáticas al estar limitadas a mostrar información permanente, privando al navegante de poder interactuar con la empresa visitada, cobran por hacer el registro de la empresa y la publicación de información.

Adicionalmente, se indica que la mayoría de los hoteles de la población de estudio venden a través de sitios Web tales como trivago.com, tripadvisor.com, booking.com, despegar.com, zonaturística.com, hotel-es.com, rumbo.es, priceline.com, expedia.mx y agoda.com., los cuales son buscadores llamados metabuscadores de hoteles, mientras que, al momento de realizarse el estudio, eran pocos los pequeños restaurantes los que hacían uso de las plataformas Uber Eats, DiDi Food o Rapid para realizar el proceso de ventas.

Para obtener la muestra de ambas poblaciones se usó la siguiente fórmula (Vázquez y Trespalacios, 2002):

$\mathrm{n}=\left(\mathrm{Z}^{2} * \mathrm{~N} * \mathrm{P}(1-\mathrm{P})\right) /\left((\mathrm{N}-1) * \mathrm{~K}^{2}+\mathrm{Z}^{2} * \mathrm{P}(1-\right.$ P) )

Donde $\mathrm{N}$ es la población total o universo de estudio, $\mathrm{n}$ es el tamaño resultante de la muestra, $\mathrm{K}$ se define como el error o máxima diferencia entre la proporción muestral y la proporción de la población que se está dispuesto a aceptar en el nivel de confianza propuesto, $\mathrm{Z}$ es el número de unidades de desviación típica en la distribución normal que producirá el grado deseado de confianza, y $\mathrm{P}$ es el porcentaje de la población que posee las características de interés, el cual se podría calcular mediante una prueba piloto, pero si no se conoce de antemano, como es este caso, se recomienda utilizar el caso más desfavorecedor de $50 \%$.

Lo0s valores considerados para la realización de este estudio son los siguientes:

$\mathrm{N}=79$ restaurantes y 97 hoteles,

$\mathrm{Z}=1.96$ para el $95 \%$,

$\mathrm{P}=0.5 \mathrm{y}$

$\mathrm{K}=0.05$

el resultado fue de 66 y 78 empresas respectivamente como muestras; se señala que durante el proceso de la aplicación del instrumento de recolección de datos se detectó que algunas empresas no estaban en la dirección indicada y en algunos casos se tuvo que hacer varias visitas, ya que el entrevistado debía ser el administrador de la empresa al considerarse que éste tiene una amplia perspectiva de las variables investigadas; $\sin$ embargo, algunos administradores no quisieron responder el cuestionario, por lo que se procedió a hacer los reemplazos correspondientes, por lo que no se vio afectado el tamaño de la muestra para efectos del estudio.

\subsection{Variables}

Los ítems que constituyen las variables estudiadas se indican en las tablas 1, 2 y 3, los cuales, como ya se mencionó, fueron construidos después de la revisión bibliográfica realizada. Para responder, se hizo uso de escalas Likert con rangos de 5 puntos, donde 1 equivale a "totalmente en desacuerdo", mientras que 5 equivale a "totalmente de acuerdo" Es importante señalar que se evitó el cuestionamiento de aspectos que se pudieran ver comprometedores (como aquellas que tuvieran que ver con dinero) con la intención de que las respuestas no se obtuvieran con sesgos, obteniendo de esta manera una mayor veracidad. Se señala que para conocer la fiabilidad de cada escala, se usó el alfa de Cronbach, alcanzándose valores satisfactorios, tomando en cuenta que valores superiores a 0.7 son tomados como suficientes, e inclusive se han llegado a considerar valores por encima de 0.6 como aceptables (Rungasamy et al., 2002).

Tabla 1. Variables del bloque Competitividad

\begin{tabular}{|c|l|c|}
\hline Nombre & \multicolumn{1}{|c|}{ Definición } & Alfa \\
\hline Conocimiento & $\begin{array}{l}\text { Ítem que mide el grado de conocimiento que se tiene sobre los conceptos de } \\
\text { competitividad dentro de la organización }\end{array}$ & - \\
\hline & $\bullet$ En nuestra empresa se conoce y domina el concepto de competitividad & \\
\hline Aspectos & $\begin{array}{l}\text { Diez ítems que reflejan la valoración de aspectos de competitividad que se } \\
\text { presentan en la empresa }\end{array}$ & 0.8279 \\
\hline & $\begin{array}{l}\text { Capacidad tecnológica } \\
\text { - Mejores costos } \\
\text { - Mejores precios }\end{array}$ & \\
\hline
\end{tabular}




\begin{tabular}{|c|c|c|}
\hline & $\begin{array}{l}\text { - Calidad de los procesos de fabricación o de prestación del servicio } \\
\text { - Rapidez y seriedad en los plazos de entrega } \\
\text { - Imagen de la empresa ante los clientes } \\
\text { - Flexibilidad para desarrollar nuevos productos y/o servicios (capacidad de } \\
\text { innovación) } \\
\text { - Capacidad de adquirir nuevos clientes (penetración de mercados) } \\
\text { - Servicio postventa y de atención al cliente } \\
\text { - Imagen de la marca de los productos/servicios ofrecidos }\end{array}$ & \\
\hline \multirow[t]{2}{*}{ Indicadores } & Siete posibles indicadores de que la empresa ha alcanzado la competitividad & 0.7543 \\
\hline & $\begin{array}{l}\text { - Se han obtenido patentes y/o licencias } \\
\text { - Se ha incrementado nuestra participación en el mercado, es decir, se ha } \\
\text { incrementado el número de clientes } \\
\text { - Se han incrementado nuestras ventas } \\
\text { - Se han ofrecido nuevos productos o se han modificado de tal manera que se } \\
\text { diferencian de los de nuestros competidores } \\
\text { - Se han mejorado notablemente nuestros procesos de producción } \\
\text { - Ha disminuido nuestra planta laboral }\end{array}$ & \\
\hline
\end{tabular}

Tabla 2. Variables del bloque Planeación estratégica

\begin{tabular}{|c|c|c|}
\hline Nombre & Definición & Alfa \\
\hline \multirow[t]{2}{*}{ Aplicación } & $\begin{array}{l}\text { Constituida por un ítem que mide el grado de aplicación que se hace de la } \\
\text { planeación estratégica dentro de la empresa. }\end{array}$ & - \\
\hline & - La planeación estratégica se lleva a cabo en la empresa & \\
\hline Actividades & $\begin{array}{l}\text { Quince ítems con los que se trata de reflejar la valoración dada a las actividades } \\
\text { relacionadas con la planeación estratégica y que son realizadas en el interior de la } \\
\text { empresa }\end{array}$ & 0.8117 \\
\hline & $\begin{array}{l}\text { - La empresa tiene definidos los objetivos estratégicos a mediano y largo plazo } \\
\text { - La misión y la visión de la empresa es conocida por todos los empleados } \\
\text { - Los conceptos de planeación estratégica son comprendidos en la organización } \\
\text { - Los directivos tienen espacios de tiempos específicos para las actividades de } \\
\text { planeación } \\
\text { - Para la definición de los objetivos y estrategias se consideran los objetivos de los } \\
\text { clientes y se analizan indicadores de previsiones económicas, demográficas, } \\
\text { políticas, tecnológicas... } \\
\text { - La empresa establece planes de desarrollo y crecimiento en nuevos mercados } \\
\text { - Se evalúan constantemente los factores internos (procesos, desempeño del } \\
\text { personal, etc.) y externos (participación de mercado, satisfacción de los clientes, } \\
\text { relaciones con proveedores, etc.) } \\
\text { - Regularmente se llevan a cabo evaluaciones de desempeño, tanto de los } \\
\text { directivos como de los empleados } \\
\text { - Las reflexiones sobre el futuro de la empresa, las amenazas del entorno y } \\
\text { oportunidades de negocio, y las líneas de actuación a seguir están documentados } \\
\text { formalmente en los planes estratégicos, planes de marketing anuales, planes de } \\
\text { ventas y/o presupuestos anuales } \\
\text { - En la empresa se manejan indicadores que permiten la evaluación y mejora } \\
\text { continua de los procesos } \\
\text { - Es utilizado algún tipo de herramienta analítica que permiten trabajar con toda la } \\
\text { información estratégica que posee la empresa (FODA, Cuadro de mando de } \\
\text { integral, etc.) } \\
\text { - Para la definición de los objetivos, así como para detectar amenazas y } \\
\text { oportunidades en nuestro entorno y puntos fuertes o áreas de mejora de la } \\
\text { empresa, se utiliza la información recabada en análisis anteriores. } \\
\text { en cuanto a capacidades, recursos, servicios, políticas, necesidades de formación, } \\
\text { etc. } \\
\text { posicionamiento en el mercado (cómo se quiere que el cliente vea a la empresa) }\end{array}$ & \\
\hline
\end{tabular}


Tabla 3. Variables del bloque Tecnologías de información

\begin{tabular}{|c|c|c|}
\hline Nombre & Definición & Alfa \\
\hline \multirow[t]{2}{*}{ Reconocimiento } & $\begin{array}{l}\text { Ítem que mide reconocimiento que se da a las TI como una herramienta } \\
\text { estratégica dentro de la organización }\end{array}$ & - \\
\hline & - En nuestra empresa se reconoce el papel estratégico que desempeñan las TI & \\
\hline \multirow[t]{2}{*}{ Aspectos } & $\begin{array}{l}\text { Cuatro ítems que reflejan la valoración de aspectos relacionados con las TI dentro } \\
\text { de la empresa }\end{array}$ & 0.8004 \\
\hline & $\begin{array}{l}\text { - La organización considera la inversión y uso de las TI en el diseño de la } \\
\text { estrategia empresarial } \\
\text { - Los empleados tienen acceso a las TI desde sus puestos de trabajo } \\
\text { - La empresa hace uso de las herramientas TI (correo electrónico, groupware, } \\
\text { Intranet o Web corporativa, grupos de noticias, software para } \\
\text { presentaciones...) para dar a conocer la misión y los valores de la organización } \\
\text { hacia el interior de la propia empresa y hacia el exterior } \\
\text { - El personal posee capacitación tecnológica y está preparado para sacar partido } \\
\text { a las TI }\end{array}$ & \\
\hline \multirow[t]{2}{*}{ Uso } & $\begin{array}{l}\text { Cuatro ítems para conocer el grado de uso dado a las TI en las actividades diarias } \\
\text { de la empresa. }\end{array}$ & 0.7287 \\
\hline & $\begin{array}{l}\text { - Se hace uso intensivo en actividades de manufactura o producción } \\
\text { - Se hace uso intensivo en procesos administrativos (contabilidad, nómina, } \\
\text { gestión bancaria, etc.) } \\
\text { - Se hace uso intensivo en relaciones con los clientes (realización de pedidos, } \\
\text { ventas, cobros, etc.) } \\
\text { - Se hace uso intensivo en relaciones con los proveedores (compras, pagos, etc.) }\end{array}$ & \\
\hline
\end{tabular}

\section{RESULTADOS}

En este apartado se muestran los resultados obtenidos en cada una de las variables estudiadas. Considerando que se hizo uso de escalas Likert con rangos de 5 puntos, puede decirse que los valores aceptables en los diferentes aspectos evaluados son de 3 a 5 . Cabe resaltar que se encontró que la vida de los hoteles estudiados se podría considerar como aceptable, ya que $67 \%$ tienen una antigüedad mayor a 10 años, lo que podría indicar la estabilidad del mercado que atienden. En el caso de los pequeños restaurantes la situación es diferente, ya que la mayoría (52\%) reportaron una vida entre 6 y 10 años, y solamente $6 \%$ indicaron una existencia superior a 10 años.

\subsection{Competitividad}

En cuanto a la competitividad, los empresarios reconocen su importancia y resaltan el conocimiento que tienen sobre su concepto y lo que implica; de hecho, esto se ve reforzado por las medias obtenidas en los aspectos competitivos que atañen a la empresa, donde se obtuvo una media promedio aceptable de 4.24, destacándose la rapidez y seriedad en los plazos de entrega, así como los mejores precios que, según los encuestados, los diferencia de sus competidores.

Sin embargo, lo anterior no se ve reflejado de manera adecuada en los indicadores competitivos de las empresas de estudio. Los hechos de no ofrecer nuevos productos (en los restaurantes) y servicios (en los hoteles), así como la percepción de que las ventas y la participación de mercado se han mantenido estables (e incluso, se percibe una disminución provocada, según la opinión de algunos de ellos, por cuestiones de la industria petrolera y de la economía nacional) indican que las empresas de estudio no muestran una competitividad satisfactoria (con medias obtenidas entre 2.90 y 3.20 ).

En este sentido, Leyva et al. (2018) destacan que toda organización debe ser consciente de que la competitividad es un tema importante que exige una gestión empresarial efectiva y muchas pequeñas empresas cuentan con debilidades de gestión.

\subsection{Planeación estratégica}

En este punto, se debe destacar la falta de un conocimiento concreto sobre los conceptos de planeación estratégica, obteniéndose en este ítem una media baja de 2.87. Incluso, algunos encuestados llegaron a comentar que en sus empresas solo se llevaba a cabo la planeación diaria de ingresos y gastos. Cabe señalar que en este aspecto no hubo diferencia notable entre los restauranteros y hoteleros.

En cuanto a las valoraciones obtenidas sobre las actividades de planeación estratégica, éstas vinieron a corroborar lo antes expuesto: no se realizan actividades de planeación estratégica dentro de las empresas estudiadas, siendo las 
actividades "Es utilizado algún tipo de herramienta analítica que permiten trabajar con toda la información estratégica que posee la empresa (FODA, Cuadro de mando de integral, etc.)", "La empresa tiene definidos los objetivos estratégicos a mediano y largo plazo" y "Para la definición de los objetivos y estrategias se consideran los objetivos de los clientes y se analizan indicadores de previsiones económicas, demográficas, políticas, tecnológicas..." las que obtienen la medias más baja $(1.64,2.05$ y 2.11 respectivamente). Se señala que se deduce que aquellas actividades que obtienen medias relativamente altas son porque se realizan de forma empírica, tal como "Los directivos tienen espacios de tiempos específicos para las actividades de planeación" (3.75).

Pese a que la literatura señala a la planeación estratégica como un factor muy importante en el desarrollo empresarial, las actividades relacionadas con la misma no son llevadas a cabo con plenitud y conciencia (hallazgo similar al de Palacio-Fierro et al. (2017) quienes encontraron en su estudio que la gran mayoría de las empresas incluyen esta planeación en sus estructuras organizativas por escrito, aunque en la práctica, la ejecución de la misma es un tema olvidado. De manera similar, Celi (2015) encontró en su estudio que las empresas no aplican planeación estratégica realizando sus actividades en forma empírica.

\subsection{Tecnologías de la información}

Este apartado se inicia destacando que $79 \%$ de los empresarios manifestaron el papel estratégico que juegan las TI dentro de la empresa (aunque no se debe pasar por alto que hubo 3 que manifestaron indiferencia en cuanto a la importancia que tienen las TI en las actividades empresariales).

Es relevante señalar que $83 \%$ de los entrevistados indicaron la necesidad de invertir y actualizar las TI, así como de hacer uso de ellas como parte de la estrategia empresarial. Sin embargo, se destaca que $72 \%$ de los encuestados otorgaron una valoración menor o igual a 3 al aspecto relacionado con el acceso a las TI por parte de los empleados desde su puesto de trabajo. De manera parecida, el aspecto "El personal posee capacitación tecnológica y está preparado para sacar partido a las TI" obtiene una media no favorable de 2.87. Similarmente, al respecto Celi (2015) destaca la ausencia de cualquier tipo de capacitación a los empleados por parte de la alta dirección empresarial. Esta falta de capacitación ha limitado la creación de nuevos conocimientos y habilidades que podrían incidir en un uso estratégico de estas herramientas.

En cuanto a los usos de las TI, los procesos administrativos (caja, contabilidad, nómina, etc.) fue el mejor valorado ya que prácticamente todos los empresarios destacaron este uso (4.78 de media). En cuanto a las relaciones con los clientes, muchos empresarios también destacaron su uso, sin embargo, se debe hacer notar, como ya se mencionó previamente, que esta relación se da a través de metabuscadores de hoteles o plataformas para el servicio de comida y no a través de páginas Web y/o redes sociales propias que se estén actualizando y que ofrezcan un verdadero acercamiento con los clientes. En cuanto al uso en actividades de manufactura o producción (donde se podrían considerar actividades como el control estratégico de inventarios, de producción de alimentos, de ocupación hotelera, entre otros) se obtienen valoraciones no satisfactorias con una media de 3.04 .

\section{CONCLUSIONES}

Para asegurar su sobrevivencia, las empresas deben diseñar estrategias para hacer frente a las condiciones actuales del mercado, marcadas por la incursión de las TI y la globalización que las empujan a un ambiente de competencia cada vez más feroz con empresas locales, regionales, nacionales e, incluso, internacionales.

Este ambiente se ha tornado muy complejo causando efectos en elementos organizacionales como las funciones administrativas, la estructura empresarial, el proceso de toma de decisiones, las tecnologías de gestión y, principalmente, en las estrategias.

En este contexto, los pequeños restaurantes y hoteles locales se ven ante una desventaja frente a las cadenas nacionales e internacionales, que por los recursos que cuentan, se encuentran bien estructuradas.

La planeación estratégica, apoyada por las TI, ofrece una fuerte posibilidad de tener éxito ante los competidores, considerando que el objetivo de ésta es que las empresas cuenten con una base que les permita enfrentar eficientemente los cambios, gestionando estas trasformaciones para continuar con buenos niveles de competitividad en el mercado que atienden.

Dado el papel que juegan los pequeños restaurantes y hoteles en la economía local, y el ambiente competitivo que se vive en la ciudad para permanecer en el gusto de los clientes, debería inferirse que estos empresarios busquen 
ser competitivos que les permita permanecer en el mercado a través del tiempo.

No obstante, los resultados encontrados parecen mostrar otra realidad. Se debe destacar que algunos de estos empresarios han logrado encontrar su propio modo que les ha permitido permanecer en el mercado, aunque no necesariamente de forma competitiva.

La planeación estratégica, pese a todas sus bondades, parece no tener mucha cabida en las empresas de estudio.

Adicionalmente, las oportunidades ofrecidas por las TI a estas empresas parece que no son aprovechadas como debiera ser y pese a que los empresarios reconocen su importancia en el logro de ventajas competitivas, se podría decir que aún existe rezago en cuanto a su adopción y uso, lo cual coincide con lo expuesto por Castellanos et al. (2016), quienes encontraron que el mismo microempresario, al no ser un ente impulsador fuerte para su empresa, provoca que el proceso de incorporación de estas tecnologías en la empresa sea aún más lento.

Estas tecnologías, al considerarse como un apoyo indiscutible en la generación de beneficios, deben convertirse en el motor de cambio para la generación de ventajas competitivas, considerando que su incursión en las actividades planeadas estratégicamente se dé de manera adecuada, ya que su importancia real radica en la forma de cómo se implementan y aplican.

Como manifiesta Castro (2001) la tecnología no se desarrolla si se administra por sí misma; es importante que se planee considerando aquellas oportunidades y amenazas identificadas en la planeación estratégica de la empresa.

Dado que no existen estudios parecidos que permitan un análisis explicativo comparativo, se sugiere la realización de estudios similares en diferentes sectores y regiones geográficas para, de este modo, poder hacer análisis comparativos que conlleven al establecimiento concreto de planes de acción. Asimismo, se propone el establecimiento de una mejor y/o mayor relación entre las empresas y los centros de investigación y educativos, así como con las entidades gubernamentales pertinentes de modo tal que se dé una capacitación adecuada que resalten los temas investigados en el desarrollo empresarial, lo que permitiría que las organizaciones sean más competitivas en un futuro próximo 


\section{REFERENCIAS}

Alarco, G. (2011). Competitividad y desarrollo. Ed. CEMTRUM. Perú.

Bueno, E. (1996). Dirección estratégica de la empresa. Metodología, técnicas y casos. Ed. Pirámide. S.A. España.

Castellanos Galeano, J. F., Loaiza, M. H., \& Cuesta Iglesias, C. A. (2016). Importancia de las TIC para la competitividad de las Pymes en Colombia. Puente, revista científica, vol. 10, No.1, pp. 93-99.

Castro, D. F. (2001). Ciencia, innovación y futuro. Ediciones Especiales. Instituto Cubano del Libro, La Habana.

Celi, S. (2015). Propuesta de un plan estratégico para el restaurant Manila de la ciudad de Loja en el periodo 2015-2019. Tesis de Licenciado en Administración de Empresas. Universidad Nacional de Loja. Ecuador. Disponible en: https://dspace.unl.edu.ec/jspui/bitstream/123456789/8520/1/Sandra\%20Yanela\%20Celi\%20Celi.pdf

Chiavenato I. (2011). Planeación estratégica: fundamentos y aplicaciones. $2^{a}$ edición. Ed. McGraw-Hill Interamericana, México.

David, F. (2007). Conceptos de administración estratégica. 11 a edición. Ed. Pearson Educación. México

García, O. A. L., \& Hernández, E. N. P. (2017). Aplicación de las tecnologías de información y comunicación en los procesos de innovación empresarial. Revisión de la literatura. I+D Revista de Investigaciones, vol. 11, no. 1, pp. 156-166.

Heredia, E. Á. (2014). Las PYMES en México: desarrollo y competitividad. Observatorio de la Economía Latinoamericana, No. 201. Disponible en: https://www.eumed.net/cursecon/ecolat/mx/2014/cooperacion.html

Hernández, R., Fernández, C., Baptista, P. (2010). Metodología de la investigación. $5^{\text {a }}$ edición, Ed. McGraw Hill. México.

Laudon, K.; Laudon, J. (2008). Sistemas de Información Gerencial, Administración de la Empresa Digital. $10^{\text {a }}$ edición, Ed. Pearson. México.

León, J. G. M., Valenzuela, A. V. (2014). Aprendizaje, innovación y gestión tecnológica en la pequeña empresa: Un estudio de las industrias metalmecánica y de tecnologías de información en Sonora. Contaduría y administración, vol. 59, No. 4, pp. 253-284.

Leyva Carreras, A. B., Cavazos Arroyo, J., Espejel Blanco, J. E. (2018). Influencia de la planeación estratégica y habilidades gerenciales como factores internos de la competitividad empresarial de las Pymes. Contaduría y administración, vol. 63, No. 3. Disponible en: http://www.scielo.org.mx/scielo.php?script=sci_arttext\&pid=S0186-10422018000400003

López, M. (2007). Planeación estratégica de tecnologías informáticas y sistemas de información. Ed. Ingeniería. Colombia.

Mandujano, J., Ramos, M., Reyes, C., Rodríguez, L. (2014). Competencias emprendedoras en MYPES del sector servicios en el estado de Tabasco, México. Universidad Juárez Autónoma de Tabasco. Disponible en:

http://www.redpilares.org/sobre-lared/Documents/CONTRERAS\%20ET\%20AL_GESTI\%C3\%93N\%20DEL\%20CONOCIMIENTO,\% 20COMPETENCIAS\%20EMPRENDEDORAS\%20Y\%20SATISFACCI\%C3\%93N.pdf

Martínez, R. M. (2013). Servicio de Hotelería. Tesis de grado de Ingeniero Comercial. Universidad Tecnológica Intercontinental. Paraguay. Disponible http://utic.edu.py/investigacion/attachments/article/74/tesis\%20completa.pdf

Palacio-Fierro, A., Mantilla-Garcés, D., Arévalo-Chávez, P. Guadalupe-Lanas, J. Cevallos-Bravo. M. (2017). Grado de utilización de los elementos integrantes de la planeación estratégica en las PYMES de Quito. Revista CienciAmérica, julio-diciembre, vol. 6, No. 2, pp.17-24.

Pizzi, A., Brunet, I. (2013). Creación de empresas, modelos de innovación y pymes. Cuadernos del CENDES, vol. 30, No. 83, pp. 53-74.

Porter, M. (1996). Ventaja competitiva. 12a edición. Ed. Continental. México.

Robbins, S. y Coulter, M. (2014). Administración. 12a edición. Ed. Pearson Educación. México.

Rodríguez, V. J. (2005). Cómo aplicar la planeación estratégica a la pequeña y mediana empresa. $5^{\text {a }}$ edición. Ed. Thompson, México.

Rungasamy, S., Antony, J., \& Ghosh, S. (2002). Critical success factors for SPC implementation in UK small and medium enterprises: some key findings from a survey. The TQM Magazine, Vol. 14 No. 4, pp. 217224

Secretaría de Turismo (SECTUR). (2016). Glosario. Hoteles y su clasificación. Disponible en: http://www.datatur.sectur.gob.mx/SitePages/Glosario.aspx

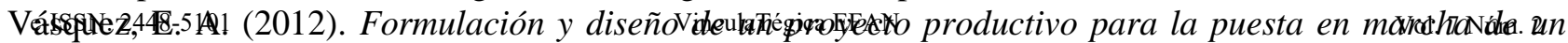


restaurante tipo gourmet en la ciudad de Cartagena. Tesis de grado de Ciencias Económicas. Universidad de Cartagena de Indias. Disponible en: http://190.25.234.130:8080/jspui/bitstream/11227/461/1/Tesis\%20de\%20grado.pdf

Vazquez, R.; Trespalacios, J.A. (2002). Marketing: estrategias y aplicaciones sectoriales. Civitas Ediciones, S.L., Madrid.

Velázquez, J. A. (2009). Diseño de un programa de capacitación para el administrador de las PYMES turísticas restauranteras del Distrito Federal. Caso: Restaurantes La Quinta. Tesis de Grado de Maestría en Ciencias en Alta Dirección de Empresas Turísticas. Escuela superior de Turismo. Sección de Estudio de Posgrado e Investigación. Instituto Politécnico Nacional. Disponible en: http://tesis.ipn.mx/bitstream/handle/123456789/8087/DISPROG.pdf?sequence=1 